\title{
$\underline{\mathbf{P}-59}$
}

\section{Evaluation of the Antiradical and Antioxidant Potential of Zingiber Zerumbet Rhizome}

\author{
Siti Balkis Budin ${ }^{1, *}$, Mohd Fuad Rahmat $\mathrm{Sam}^{2}$, Asmah Hamid ${ }^{1}$ and Ahmad Rohi Ghazali ${ }^{1}$ \\ ${ }^{1}$ Programme of Biomedical Science, School of Diagnostic and Applied Health Sciences, Faculty of Health Sciences, \\ UniversitiKebangsaan Malaysia, Jalan Raja Muda Abdul Aziz, 50300 Kuala Lumpur, Malaysia; ${ }^{2}$ Department of \\ Biomedical Science, Kulliyyah of Allied Health Sciences, International Islamic University Malaysia, Jalan Istana, 25200 \\ Kuantan, Pahang, Malaysia; E-mail: balkis6466@yahoo.com.my
}

Rhizomes of Zingiber zerumbet that belonging to genus Zingiberaceae have been used in traditional medicine, especially among the Asian community. This study aimed to investigate antioxidant potential of ethyl acetate extract of $Z$. zerumbet. Identification of extract compound rhizomes of $Z$. zerumbet was carried out by Gas Chromatography-Mass Spectrometry (GCMS). The GC-MS analysis revealed 12 compounds and the major constituent is Zerumbone (51.57\%). Determination of safe concentration Z. zerumbet towards rat red blood cells (RBCs) has been done and showed that incubated RBCs at a concentration $50 \mu \mathrm{g} / \mathrm{ml}, 25 \mu \mathrm{g} / \mathrm{ml}, 12.5 \mu \mathrm{g} / \mathrm{ml}$ and $6.25 \mu \mathrm{g} / \mathrm{ml}$ of ethyl acetate extract of $Z$. zerumbet for 1 hour not cause significant differences in hemolysis and the level of malondialdehyde (MDA) compared to the control group, $\mathrm{NaCl}$. The antioxidant properties of ethyl acetate extract of $Z$. zerumbet were compared using in vitro antioxidant methods such as DPPH radical scavenging and FRAP assays while the amount of major phenolic compounds were determined by spectrophotometric methods. The results showed that ethyl acetate extract of $Z$. zerumbet exhibited antioxidant and radical-scavenging activities at different magnitudes of potency although the effects were small to that BHT. The results obtained in the present study indicates that the rhizomes of $Z$. zerumbet at concentration range $6.25 \mu \mathrm{g} / \mathrm{ml}-50 \mu \mathrm{g} / \mathrm{ml}$ are a potential source of natural antioxidants on rat RBCs.

Keywords: Antioxidant activity, Red blood cells, Zingiber zerumbet, FRAP, DPPH. 